PROCEEDINGS OF THE

AMERICAN MATHEMATICAL SOCIETY

Volume 126, Number 1, January 1998, Pages 135-144

S 0002-9939(98)04400-1

\title{
PARAEXPONENTIALS, MUCKENHOUPT WEIGHTS, AND RESOLVENTS OF PARAPRODUCTS
}

\author{
MARÍA C. PEREYRA AND LESLEY A. WARD
}

(Communicated by J. Marshall Ash)

\begin{abstract}
We analyze the stability of Muckenhoupt's $\mathbf{R} \mathbf{H}_{\mathbf{p}}^{\mathbf{d}}$ and $\mathbf{A}_{\mathbf{p}}^{\mathbf{d}}$ classes of weights under a nonlinear operation, the $\lambda$-operation. We prove that the dyadic doubling reverse Hölder classes $\mathbf{R} \mathbf{H}_{\mathbf{p}}^{\mathbf{d}}$ are not preserved under the $\lambda$ operation, but the dyadic doubling $A_{p}$ classes $\mathbf{A}_{\mathbf{p}}^{\mathbf{d}}$ are preserved for $0 \leq \lambda \leq 1$. We give an application to the structure of resolvent sets of dyadic paraproduct operators.
\end{abstract}

\section{INTRODUCTION}

The Muckenhoupt classes of weights consist of positive locally integrable functions satisfying certain integrability conditions on intervals; see Section 2. They arise, for instance, in connection with the boundedness of the Hilbert transform and maximal function operators on $L^{p}$ spaces.

Weights in the Muckenhoupt classes are often represented as exponentials of functions in BMO; see [GC-RF]. For weights $\omega(x)$ defined on an interval $J$, with mean value one on $J$, a different representation, sometimes called the paraexponential, was introduced in $[\mathrm{FKP}]$. The correspondence is realized by an infinite product, namely:

$$
\omega(x)=\prod_{I \in \mathcal{D}(J)}\left(1+b_{I} h_{I}(x)\right), \quad b_{I}=\frac{\left\langle\omega, h_{I}\right\rangle}{m_{I} \omega} ;
$$

where $\mathcal{D}(J)$ denotes the intervals in the dyadic decomposition of the interval $J$, $\left\{h_{I}\right\}_{I \in \mathcal{D}(J)}$ are Haar functions, $\langle\cdot, \cdot\rangle$ is the scalar product in $L^{2}$, and $m_{I} \omega$ is the mean value of $\omega$ on $I$.

It was proved in $[\mathrm{FKP}]$ that if there is an $\varepsilon>0$ such that $\left|b_{I} h_{I}\right|<1-\varepsilon$ for all $I \in$ $\mathcal{D}(J)$, then the partial products converge to a weight in dyadic doubling $\mathbf{A}_{\infty}^{\mathbf{d}}$ if and only if $b=\sum_{I \in \mathcal{D}(J)} b_{I} h_{I}$ is a function of dyadic bounded mean oscillation, $\mathbf{B M O}$. A larger dictionary relating properties of $\omega$ and $b$ can be found in [B]. In particular the dyadic doubling Muckenhoupt $\mathbf{R} \mathbf{H}_{\mathbf{p}}^{\mathbf{d}}$ and $\mathbf{A}_{\mathbf{p}}^{\mathbf{d}}$ classes can be characterized by summation conditions on $b$.

Received by the editors May 9, 1996.

1991 Mathematics Subject Classification. Primary 42B99; Secondary 47A10.

Key words and phrases. Muckenhoupt weights, reverse Hölder $R H_{p}, A_{p}$, doubling weights, dyadic paraproducts, paraexponentials.

This research was supported in part by (CP) NSF grant \#DMS-93-04580 and (LW) at MSRI by NSF grant \#DMS-90-22140.

(C) 1998 American Mathematical Society 
We will consider only weights $\omega$ defined on an interval $J$, with mean value one on $J$. For such a weight we have, at least formally, the product representation (1.1). We are interested in the effect of multiplying the coefficient $b_{I}$ in each factor of the product by the same number $\lambda$. Define

$$
\omega_{\lambda}(x)=\prod_{I \in \mathcal{D}(J)}\left(1+\lambda b_{I} h_{I}(x)\right) .
$$

We call the mapping that sends $\omega \mapsto \omega_{\lambda}$ the $\lambda$-operation.

To guarantee the convergence of the products we will only consider the case $-1 \leq \lambda \leq 1$. If $\omega$ is a weight in $\mathbf{A}_{\infty}^{\mathbf{d}}$ and $-1 \leq \lambda \leq 1$, then $\omega_{\lambda}$ is also in $\mathbf{A}_{\infty}^{\mathbf{d}}$; see $[\mathrm{FKP}]$.

The $\lambda$-operation seems, at first sight, very similar to taking a $\lambda$ power of the weight, $\omega^{\lambda}$. The first two terms in the Taylor expansion of $\omega^{\lambda}=e^{\lambda b}$ coincide with the first two terms obtained by expanding the infinite product in (1.2), but the third terms differ. Both the $\mathbf{R H}_{\mathbf{p}}^{\mathbf{d}}$ and the $\mathbf{A}_{\mathbf{p}}^{\mathbf{d}}$ classes are preserved under taking powers $\omega \mapsto \omega^{\lambda}$, for $0 \leq \lambda \leq 1$.

Question. Are the $\mathbf{R} \mathbf{H}_{\mathbf{p}}^{\mathbf{d}}$ and $\mathbf{A}_{\mathbf{p}}^{\mathbf{d}}$ classes preserved under the $\lambda$-operation?

The answer is negative for the $\mathbf{R} \mathbf{H}_{\mathbf{p}}^{\mathbf{d}}$ classes, and positive for the $\mathbf{A}_{\mathbf{p}}^{\mathbf{d}}$ classes.

Theorem 1.1. For each $p>1$, there exist a weight $\omega$ in $\mathbf{R H}_{\mathbf{p}}^{\mathbf{d}}$ and a number $\lambda \in(0,1)$ such that $\omega_{\lambda}$ is not in $\mathbf{R H}_{\mathbf{p}}^{\mathbf{d}}$.

For $p>(1-\log 2)^{-1}>1$, examples with positive $\lambda$ 's are given in [P2]. In this paper we give examples with positive $\lambda$ 's for all $p>1$. Examples for all $p>1$, with $-1 \leq \lambda<0$, are given in [P1].

A related result in [P2] is that if $\omega \in \mathbf{R H}_{\mathbf{p}}^{\mathbf{d}}$, then there is a $q>1$ such that $\omega_{\lambda} \in \mathbf{R H}_{\mathbf{q}}^{\mathbf{d}}$ for all $-1 \leq \lambda \leq 1$.

Theorem 1.2. Take any $p$ and $\lambda$ such that $1 \leq p \leq \infty$ and $0 \leq \lambda \leq 1$. If the weight $\omega$ is in $\mathbf{A}_{\mathbf{p}}^{\mathbf{d}}$, then the weight $\omega_{\lambda}$ is also in $\mathbf{A}_{\mathbf{p}}^{\mathbf{d}}$.

However, the $\mathbf{A}_{\mathbf{p}}^{\mathbf{d}}$ spaces for $1 \leq p<\infty$ are not preserved by the $\lambda$-operation for negative $\lambda$; this can be shown by examples like those in [P1].

The $\lambda$-operation appears naturally in the study of the resolvents of the dyadic paraproduct [P2]. The dyadic paraproduct is a bilinear operator that appears in different guises in harmonic analysis, often replacing the ordinary product $[\mathrm{M}],[\mathrm{Ch}]$, [D]. The dyadic paraproduct $\pi_{b}$ associated to a function $b$ is defined by

$$
\pi_{b} f(x)=\sum_{I \in \mathcal{D}(J)} m_{I} f b_{I} h_{I}(x),
$$

where $m_{I} f$ is the mean value of $f$ on the interval $I$, and $b_{I}=\left\langle b, h_{I}\right\rangle$. The paraproduct $\pi_{b}$ is bounded on $L^{p}(J)$ if and only if $b \in \mathbf{B M O}^{\mathbf{d}}$. The conditions on $b$ that guarantee the existence of a bounded inverse of $\left(I-\lambda \pi_{b}\right)$ on $L_{o}^{p}(J)=\left\{f \in L^{p}(J): \int_{J} f=0\right\}$ are described in terms of properties of the weight $\omega_{\lambda}$. For doubling weights, the conditions reduce to $\omega_{\lambda} \in \mathbf{R} \mathbf{H}_{\mathbf{p}}^{\mathrm{d}}$; see [P1]. More precisely:

Theorem $1.3(\mathrm{P})$. Let $b$ be a function in $\mathbf{B M O}^{\mathrm{d}}$, take $\epsilon>0$, and let $\lambda$ be a real number such that $\left|\lambda b_{I} h_{I}\right| \leq 1-\epsilon$ for all $I \in \mathcal{D}(J)$, where $b_{I}=\left\langle b, h_{I}\right\rangle$. Then 
$\left(I-\lambda \pi_{b}\right)^{-1}$ exists and is bounded on $L_{o}^{p}(J)$ if and only if the weight $\omega_{\lambda}(x)=$ $\prod_{I \in \mathcal{D}(J)}\left(1+\lambda b_{I} h_{I}(x)\right)$ is in $\mathbf{R H}_{\mathbf{p}}^{\mathbf{d}}$.

Question. If $\left(I-\lambda \pi_{b}\right)^{-1}$ exists and is bounded on $L_{0}^{p}$ for $\lambda=1$, is the same true for all $\lambda \in[0,1]$ ?

Since the resolvent set of $\pi_{b}$ is open, there are neighborhoods of $\lambda=0$ and $\lambda=1$ for which this holds. By the discussion above, the question is equivalent to asking whether the $\mathbf{R H}_{\mathbf{p}}^{\mathbf{d}}$ classes are preserved under the $\lambda$-operation. Theorem 1.1 shows that the answer is negative. Rephrasing the theorem, we have:

Theorem 1.4. For each $p>1$, there exist a number $\lambda \in(0,1)$, a function $b \in$ $\mathbf{B M O}^{\mathbf{d}}$, and a number $\epsilon>0$ such that $\left|b_{I} h_{I}\right| \leq 1-\epsilon$ for all $I \in \mathcal{D}(J)$, the operator $\left(I-\pi_{b}\right)^{-1}$ exists and is a bounded operator on $L_{o}^{p}(J)$, but the operator $\left(I-\lambda \pi_{b}\right)$ is not invertible as an operator from $L_{o}^{p}(J)$ into $L_{o}^{p}(J)$.

In Section 2 we give notation, definitions, and some preparatory lemmas. In Section 3 we prove Theorem 1.2, and in Section 4 we construct the examples which establish Theorem 1.1.

As is customary, $C$ denotes a constant that may change from line to line.

\section{Preliminaries}

In this section we introduce the dyadic intervals and the Haar basis; and we define dyadic $\mathbf{B M O}^{\mathbf{d}}$ in terms of a Carleson condition. Next we define the dyadic doubling Muckenhoupt classes $\mathbf{A}_{\infty}^{\mathbf{d}}, \mathbf{R} \mathbf{H}_{\mathbf{p}}^{\mathbf{d}}$, and $\mathbf{A}_{\mathbf{p}}^{\mathbf{d}}$; and we recall some of their properties. We state the Fefferman-Kenig-Pipher Product Representation Theorem for $\mathbf{A}_{\infty}^{\mathbf{d}}$ weights and Buckley's Theorem characterizing $\mathbf{A}_{\mathbf{p}}^{\mathbf{d}}$ weights via summation conditions. Finally we define the $\lambda$-operation, and give a convexity lemma used in the proof of Theorem 1.2.

Let $\mathcal{D}$ denote the family of all dyadic subintervals of $[0,1]$, in other words all intervals of the form $\left(j 2^{-k},(j+1) 2^{-k}\right], j, k$ integers, $0 \leq k, 0 \leq j \leq 2^{k}-1$. Given any interval $J, \mathcal{D}(J)$ denotes the family of dyadic subintervals of $J$. Given an interval $J$, we denote its left and right halves respectively by $J_{l}$ and $J_{r}$. An interval $\widetilde{I}$ is the parent of an interval $I$ if $I$ is $\widetilde{I}_{l}$ or $\widetilde{I}_{r}$.

The Haar function associated to an interval $I$ is given by

$$
h_{I}(x)=|I|^{-1 / 2}\left(\chi_{I_{r}}(x)-\chi_{I_{l}}(x)\right) ;
$$

here $\chi_{I}$ denotes the characteristic function of the interval $I$. The set of Haar functions indexed by $\mathcal{D}(J)$ forms a basis of $L_{o}^{2}(J)=\left\{f \in L^{2}(J): \int_{J} f=0\right\}$; see $[\mathrm{H}]$.

A locally integrable function $b$ on $[0,1]$ is in the space of dyadic bounded mean oscillation $\mathbf{B M O}^{\mathbf{d}}$ if there is a constant $C$ such that $\int_{J}\left|b(x)-m_{J} b\right|^{2} d x \leq C|J|$ for all $J \in \mathcal{D}$, where $m_{J} b=\frac{1}{|J|} \int_{J} b$. The function $b$ is in $\mathbf{B M O} \mathbf{d}^{\mathbf{d}}$ if and only if the Carleson condition on the Haar coefficients $b_{I}=\left\langle b, h_{I}\right\rangle$ of $b$ holds: $\sum_{I \in \mathcal{D}(J)} b_{I}^{2} \leq$ $C|J|$ for all $J \in \mathcal{D}$, with a constant $C$ independent of $J$; see [Ch], [M].

2.1. Dyadic weights. We consider weights defined on the interval $J_{0}=[0,1]$.

A dyadic doubling weight $\omega$ is a positive locally integrable function such that $\int_{\widetilde{I}} \omega \leq C \int_{I} \omega$ for all intervals $I \in \mathcal{D}$, where $\widetilde{I}$ is the parent of $I$ and $C$ is a constant independent of $I$. 
A weight $\omega$ is in the dyadic doubling $A_{\infty}$ class $\mathbf{A}_{\infty}^{\mathbf{d}}$ if $\omega$ is dyadic doubling, and there is a constant $C$ such that $\frac{1}{|I|} \int_{I} \omega \leq C \exp \left(\frac{1}{|I|} \int_{I} \log \omega\right)$, for all $I \in \mathcal{D}$.

A weight $\omega$ is in the dyadic doubling reverse Hölder class $\mathbf{R H}_{\mathbf{p}}^{\mathbf{d}}$, for $1<p<\infty$, if $\omega$ is dyadic doubling, and there is a constant $C$ such that $\left(\frac{1}{|I|} \int_{I} \omega^{p}\right)^{1 / p} \leq C \frac{1}{|I|} \int_{I} \omega$, for all $I \in \mathcal{D}$.

A weight $\omega$ is in the dyadic doubling $A_{p}$ class $\mathbf{A}_{\mathbf{p}}^{\mathbf{d}}$, for $1<p<\infty$, if $\omega$ is dyadic doubling, and there is a constant $C$ such that $\left(\frac{1}{|I|} \int_{I} \omega\right)\left(\frac{1}{|I|} \int_{I} \omega^{-1 /(p-1)}\right)^{p-1} \leq C$ for all $I \in \mathcal{D}$.

A weight $\omega$ is in the dyadic doubling $A_{1}$ class $\mathbf{A}_{1}^{\mathrm{d}}$ if $\omega$ is dyadic doubling, and there is a constant $C$ such that $\frac{1}{|I|} \int_{I} \omega \leq C \omega(x)$ for a.e. $x \in I$, for all $I \in \mathcal{D}$.

The canonical examples are $\omega(x)=|x|^{\alpha}$. In this case $\omega \in \mathbf{A}_{\infty}^{\mathbf{d}}$ if and only if $\alpha>-1, \omega \in \mathbf{R H}_{\mathbf{p}}^{\mathbf{d}}$ if and only if $\alpha>-1 / p$, for $1<p<\infty, \omega \in \mathbf{A}_{\mathbf{p}}^{\mathbf{d}}$ if and only if $-1<\alpha<p-1$, and $\omega \in \mathbf{A}_{1}^{\mathbf{d}}$ if and only if $-1<\alpha \leq 0$.

The class $\mathbf{A}_{\infty}^{\mathbf{d}}$ is the union of the $\mathbf{R H}_{\mathbf{p}}^{\mathbf{d}}$ classes, and also of the $\mathbf{A}_{\mathbf{p}}^{\mathbf{d}}$ classes. The class $\mathbf{A}_{1}^{\mathbf{d}}$ is strictly contained in the intersection of the $\mathbf{A}_{\mathbf{p}}^{\mathbf{d}}$ classes. More precisely:

$$
\mathbf{A}_{\infty}^{\mathrm{d}}=\bigcup_{\mathbf{p}>1} \mathbf{R} \mathbf{H}_{\mathbf{p}}^{\mathrm{d}}=\bigcup_{\mathbf{p}>1} \mathbf{A}_{\mathbf{p}}^{\mathrm{d}}, \quad \mathbf{A}_{1}^{\mathrm{d}} \subset \bigcap_{\mathbf{p}>1} \mathbf{A}_{\mathbf{p}}^{\mathrm{d}} .
$$

These properties are known for weights that satisfy the conditions above for all intervals $I$; see [GC-RF]. In our case we consider only dyadic intervals, but we also assume that the weights are dyadic doubling, which yields (2.1); see [B]. An explicit example of a function in $\bigcap_{p>1} \mathbf{A}_{\mathbf{p}}^{\mathbf{d}}$ but not in $\mathbf{A}_{\mathbf{1}}^{\mathbf{d}}$ can be found in [JN].

To each weight $\omega \in \mathbf{A}_{\infty}^{\mathbf{d}}$, with mean value one on $J_{0}$, we associate a function $b=b_{\omega}$ so that

$$
\omega(x)=\prod_{I \in \mathcal{D}}\left(1+b_{I} h_{I}(x)\right), \quad b_{I}=\frac{\left\langle\omega, h_{I}\right\rangle}{m_{I} \omega},
$$

and

$$
b(x)=\sum_{I \in \mathcal{D}} b_{I} h_{I}(x) .
$$

Theorem 2.1 (R. Fefferman, Kenig, Pipher). Let $\left\{b_{I}\right\}_{I \in \mathcal{D}\left(J_{0}\right)}$ and $\epsilon>0$ be given, with $\left|b_{I} h_{I}\right| \leq 1-\epsilon$ for all $I \in \mathcal{D}$. Then the product (2.2) belongs to $\mathbf{A}_{\infty}^{\mathbf{d}}$ if and only if $b \in \mathbf{B M O}^{\mathbf{d}}$, that is, if and only if there is a constant $C$ such that

$$
\sum_{I \in \mathcal{D}(J)} b_{I}^{2} \leq C|J|, \quad \forall J \in \mathcal{D} .
$$

If so, the weight $\omega$ is a dyadic doubling weight.

This is proved in $[\mathrm{FKP}]$.

The dyadic $\mathbf{R} \mathbf{H}_{\mathbf{p}}^{\mathbf{d}}$ and $\mathbf{A}_{\mathbf{p}}^{\mathbf{d}}$ classes can also be characterized by summation conditions. Part (a) of the next theorem, and related results, appear in [B].

Theorem 2.2. Let $\omega$ be a dyadic doubling weight.

(a) (Buckley) $\omega \in \mathbf{A}_{\mathbf{p}}^{\mathbf{d}}, 1<p<\infty$, if and only if there is a constant $C$ such that

$$
\sum_{I \in \mathcal{D}(J)}\left(\frac{m_{I} \omega}{m_{J} \omega}\right)^{\frac{-1}{p-1}} b_{I}^{2} \leq C|J|, \quad \forall J \in \mathcal{D} .
$$


(b) $\omega \in \mathbf{A}_{1}^{\mathbf{d}}$ if and only if there is a constant $C$ such that

$$
\frac{m_{J} \omega}{m_{I} \omega} \leq C, \quad \forall I \in \mathcal{D}(J), \quad \forall J \in \mathcal{D} .
$$

Proof of $(b)$. $(\Rightarrow)$ Integrate over $I \in \mathcal{D}(J)$ in the definition of $\mathbf{A}_{1}^{\mathbf{d}} . \quad(\Leftarrow)$ By the Lebesgue differentiation theorem, the limit of $m_{I} \omega$, as a sequence of intervals $I \in$ $\mathcal{D}(J)$ shrinks to a point $x \in J$, is $\omega(x)$ for almost every $x$.

Let $\widetilde{I}$ be the parent of $I$. Denote by $s_{I}$ the proportion of the mass of $\widetilde{I}$ that is carried by $I$. Then

$$
2 s_{I}=\frac{m_{I} \omega}{m_{\widetilde{I}} \omega}=1+b_{\widetilde{I}} h_{\widetilde{I}}(x), \quad \forall x \in I .
$$

2.2. The $\lambda$-operation. The nonlinear operation that sends the weight $\omega$ given by $\omega=\prod_{I \in \mathcal{D}}\left(1+b_{I} h_{I}\right)$ into the weight $\omega_{\lambda}$,

$$
\omega \mapsto \omega_{\lambda}=\prod_{I \in \mathcal{D}}\left(1+\lambda b_{I} h_{I}\right)
$$

is called the $\lambda$-operation.

Remark 2.3. Dyadic doubling and $\mathbf{A}_{\infty}^{\mathbf{d}}$ weights are preserved under the $\lambda$-operation for $-1 \leq \lambda \leq 1$. This is a consequence of Theorem 2.1, and the observation that since $\left|\lambda \bar{b}_{I}\right| \leq\left|b_{I}\right|, b \in \mathbf{B M O}^{\mathbf{d}}$ implies $\lambda b \in \mathbf{B M O}^{\mathbf{d}}$; see [P2].

To understand how the $\lambda$-operation affects $\mathbf{R} \mathbf{H}_{\mathbf{p}}^{\mathbf{d}}$ and $\mathbf{A}_{\mathbf{p}}^{\mathbf{d}}$ weights, let us first consider how it affects quotients of mean values of $\omega$ over consecutive nested intervals. Accordingly define

$$
s_{I}(\lambda)=\frac{m_{I} \omega_{\lambda}}{2 m_{\widetilde{I}} \omega_{\lambda}} .
$$

The $\lambda$-operation is a nonlinear operation on the weight, but it is linear at the level of the $s_{I}$ 's:

Lemma 2.4. $s_{I}(\lambda)=\frac{1}{2}+\lambda\left(s_{I}-\frac{1}{2}\right)$.

Proof. This is an immediate consequence of (2.4).

Lemma 2.5. Let $a_{1}, \ldots, a_{n}$ be positive numbers. Let $a_{j}(\lambda)=1+\lambda\left(a_{j}-1\right)$, and $A_{n}(\lambda)=\prod_{j=1}^{n} a_{j}(\lambda)$. Then

$$
A_{n}(\lambda) \geq \min \left\{1, A_{n}(1)\right\} .
$$

Proof. On expanding the product we get

$$
A_{n}(\lambda)=\prod_{k=1}^{n}\left((1-\lambda)+\lambda a_{k}\right)=\sum_{i=0}^{n}(1-\lambda)^{n-i} \lambda^{i} \sum_{k_{1} \neq \ldots \neq k_{i}} \prod_{j=1}^{i} a_{k_{j}},
$$

where the index notation $k_{1} \neq \ldots \neq k_{i}$ means that the $k_{j}$ 's are pairwise different, and $1 \leq k_{j} \leq n$. Now, using repeatedly the fact that for positive numbers the arithmetic mean is at least the geometric mean, we find that

$$
\sum_{k_{1} \neq \ldots \neq k_{i}} \prod_{j=1}^{i} a_{k_{j}} \geq N_{n, i}\left(\prod_{k_{1} \neq \ldots \neq k_{i}} \prod_{j=1}^{i} a_{k_{j}}\right)^{1 / N_{n, i}},
$$

where $N_{n, i}$ is the number of ways one can choose $i$ numbers from a collection of $n$ numbers, that is, $N_{n, i}=\frac{n !}{i !(n-i) !}$. Observe that in the double product in (2.9), each 
$a_{j}$ occurs as many times as there are possible ways to choose $i-1$ numbers from a collection of $n-1$ numbers, that is, $N_{n-1, i-1}$. Since $N_{n-1, i-1} / N_{n, i}=i / n$, the right hand side of $(2.9)$ is equal to

$$
N_{n, i}\left(\prod_{j=1}^{n} a_{j}\right)^{i / n}=N_{n, i}\left(A_{n}(1)\right)^{i / n} .
$$

We conclude that

$$
\begin{aligned}
A_{n}(\lambda) & \geq \sum_{i=0}^{n}(1-\lambda)^{n-i} \lambda^{i} N_{n, i}\left(A_{n}(1)\right)^{i / n} \\
& =\left(\lambda\left(A_{n}(1)\right)^{1 / n}+(1-\lambda)\right)^{n} .
\end{aligned}
$$

Fix $A \geq 0$. The function $f(\lambda)=(\lambda A+(1-\lambda))^{n}$ is monotonic for $\lambda \in[0,1]$; hence it is bounded below by $\min \{f(0), f(1)\}$. Set $A=\left(A_{n}(1)\right)^{1 / n}$; then $f(0)=1$, $f(1)=A_{n}(1)$. This completes the proof of the lemma.

\section{The theorem for $\mathbf{A}_{\mathbf{p}}^{\mathbf{d}}$ Weights}

Theorem 3.1. Take any $p$ and $\lambda$ such that $1 \leq p \leq \infty$ and $0 \leq \lambda \leq 1$. If the weight $\omega$ is in $\mathbf{A}_{\mathbf{p}}^{\mathbf{d}}$, then the weight $\omega_{\lambda}$ is also in $\mathbf{A}_{\mathbf{p}}^{\mathbf{d}}$.

Proof. By Remark $2.3, \omega_{\lambda}$ is a dyadic doubling weight, and $\omega \in \mathbf{A}_{\infty}^{\mathbf{d}}$ implies $\omega_{\lambda} \in$ $\mathbf{A}_{\infty}^{\mathrm{d}}$.

For dyadic intervals $I \subset J$, the ratios $\frac{m_{I} \omega}{m_{J} \omega}$ and $\frac{m_{I} \omega_{\lambda}}{m_{J} \omega_{\lambda}}$ can be explicitly computed as products of $s_{K}$ 's and $s_{K}(\lambda)$ 's respectively:

$$
\frac{m_{I} \omega}{m_{J} \omega}=\prod_{I \subseteq K \subset J} 2 s_{K}, \quad \text { and } \quad \frac{m_{I} \omega_{\lambda}}{m_{J} \omega_{\lambda}}=\prod_{I \subseteq K \subset J} 2 s_{K}(\lambda),
$$

where $K \in \mathcal{D}(J), 2 s_{K}(\lambda)=1+\lambda\left(2 s_{K}-1\right)$, and $2 s_{K}=m_{K} \omega / m_{\widetilde{K}} \omega$.

These are the kind of products considered in Lemma 2.5. Since $\omega$ is dyadic doubling, (2.4) shows that $\epsilon \leq 2 s_{K} \leq 2-\epsilon$. In particular, $a_{K}=2 s_{K}>0$. We can apply Lemma 2.5 to each pair $(I, J)$ of dyadic intervals, $I \subset J$, where $n=\log _{2}(|J| /|I|), A_{n}(\lambda)=m_{I} \omega_{\lambda} / m_{J} \omega_{\lambda}$, and $A_{n}(1)=m_{I} \omega / m_{J} \omega$, to conclude that

$$
\left(\frac{m_{I} \omega_{\lambda}}{m_{J} \omega_{\lambda}}\right)^{r} \leq \max \left\{1,\left(\frac{m_{I} \omega}{m_{J} \omega}\right)^{r}\right\}
$$

for all negative $r$.

Case $1<p<\infty$. By Theorem 2.2(a), it suffices to find a constant $C$ such that

$$
\frac{1}{|J|} \sum_{I \in \mathcal{D}(J)}\left(\frac{m_{I} \omega_{\lambda}}{m_{J} \omega_{\lambda}}\right)^{\frac{-1}{p-1}} b_{I, \lambda}^{2} \leq C \quad \forall J \in \mathcal{D},
$$

where $b_{I, \lambda}=\left\langle\lambda b, h_{I}\right\rangle=\lambda b_{I}$. 
We use (3.2), with $r=-1 /(p-1)$, to estimate the left hand side of (3.3). Using the observation that $b_{I, \lambda}^{2} \leq b_{I}^{2}$ for $0<\lambda<1$, we get

$$
\begin{aligned}
\frac{1}{|J|} \sum_{I \in \mathcal{D}(J)}\left(\frac{m_{I} \omega_{\lambda}}{m_{J} \omega_{\lambda}}\right)^{\frac{-1}{p-1}} b_{I, \lambda}^{2} & \leq \frac{1}{|J|} \sum_{I \in \mathcal{D}(J)} \max \left\{1,\left(\frac{m_{I} \omega}{m_{J} \omega}\right)^{\frac{-1}{p-1}}\right\} b_{I}^{2} \\
& \leq \frac{1}{|J|} \sum_{I \in \mathcal{D}(J)} b_{I}^{2}+\frac{1}{|J|} \sum_{I \in \mathcal{D}(J)}\left(\frac{m_{I} \omega}{m_{J} \omega}\right)^{\frac{-1}{p-1}} b_{I}^{2} \leq C ;
\end{aligned}
$$

where $C$ is a constant independent of the interval $J$. The last inequality holds since $\omega \in \mathbf{A}_{\mathbf{p}}^{\mathbf{d}} \subset \mathbf{A}_{\infty}^{\mathbf{d}}$, by Theorems 2.1 and $2.2(\mathrm{a})$.

Case $p=1$. By Theorem 2.2(b), it suffices to find a constant $C$ such that

$$
\left(\frac{m_{I} \omega_{\lambda}}{m_{J} \omega_{\lambda}}\right)^{-1} \leq C, \quad \forall I \in \mathcal{D}(J), \quad \forall J \in \mathcal{D} .
$$

We use (3.2) again, with $r=-1$, to conclude that the left hand side of (3.4) is bounded by $\max \left\{1,\left(\frac{m_{I} w}{m_{J} w}\right)^{-1}\right\}$, which in turn is bounded because $\omega \in \mathbf{A}_{\mathbf{1}}^{\mathbf{d}}$.

\section{EXAMPLES IN $\mathbf{R} \mathbf{H}_{\mathbf{p}}^{\mathbf{d}}$}

Theorem 4.1. For each $p>1$, there exist a dyadic doubling weight $\omega$ in $\mathbf{R H}_{\mathbf{p}}^{\mathbf{d}}$ and a number $\lambda \in(0,1)$ such that $\omega_{\lambda}$ is not in $\mathbf{R} \mathbf{H}_{\mathbf{p}}^{\mathbf{d}}$.

Proof. Our examples are of the following form. Let $I_{i}=\left[2^{-i}, 2^{-i+1}\right]$ for $i \geq 1$, and $J_{i}=\left[0,2^{-i}\right]$ for $i \geq 0$. Fix numbers $s_{i} \in(0,1)$ for $i \geq 1$. For $x \in[0,1]$ let

$$
\omega(x)=\sum_{i=1}^{\infty} c_{i} \chi_{I_{i}}(x), \quad \text { where } \quad c_{i}=2^{i} s_{1} \ldots s_{i-1}\left(1-s_{i}\right) .
$$

One can think of $\omega$ as the weight of mass one obtained by assigning the fractions $s_{i}$ and $1-s_{i}$ of the mass of $J_{i-1}$ to the left half $J_{i}$ and the right half $I_{i}$ respectively of $J_{i-1}$, for each $i \geq 1$. In the notation of Section 2 ,

$$
s_{i}=s_{J_{i}}=\frac{m_{J_{i}} \omega}{2 m_{J_{i-1}} \omega}, \quad \text { and } \quad m_{J_{i}} \omega=2^{i} s_{1} \ldots s_{i-1} s_{i} .
$$

We further assume that the sequence $\left\{s_{i}\right\}$ is $n$-periodic: there is a smallest positive integer $n$ such that $s_{i+n}=s_{i}$ for all $i \geq 1$. Such an $\omega$ is a dyadic doubling weight. For $n$-periodic weights of this form,

$$
c_{k n+j}=\left(2^{n} s_{1} \ldots s_{n}\right)^{k} c_{j}, \quad \forall k \geq 0, j \geq 1 .
$$

Claim. The $\mathbf{R H}_{\mathbf{p}}^{\mathbf{d}}$ condition $\frac{1}{|I|} \int_{I} \omega^{p} \leq C\left(\frac{1}{|I|} \int_{I} \omega\right)^{p}$, for all $I \in \mathcal{D}$, for an $n$ periodic weight of the form (4.1) reduces to the condition

$$
2^{n} s_{1} \ldots s_{n}<2^{n / p}
$$

To see this, first note that $\omega$ is constant on each dyadic interval $I$ whose left endpoint is not 0 , so the reverse Hölder $p$ condition holds with $C=1$ on these intervals. The only other dyadic intervals are $J_{l}=\left[0,2^{-l}\right], l \geq 0$. 
Write $l=q n+m$, where $q$ and $m$ are non-negative integers and $0 \leq m \leq n-1$. We use (4.3) to compute the mean of $\omega^{p}$ on $J_{l}=\bigcup_{i=l+1}^{\infty} I_{i}$ :

$$
\begin{aligned}
\frac{1}{\left|J_{l}\right|} \int_{J_{l}} \omega^{p} & =2^{l} \sum_{i=l+1}^{\infty} \int_{I_{i}} \omega^{p}=2^{l} \sum_{i=q n+m+1}^{\infty} c_{i}^{p} 2^{-i} \\
& =2^{l} \sum_{k=q}^{\infty} \sum_{j=1}^{n}\left(c_{k n+j+m}\right)^{p} 2^{-(k n+j+m)} \\
& =2^{l} \sum_{k=q}^{\infty} \sum_{j=1}^{n}\left(2^{n} s_{1} \ldots s_{n}\right)^{k p}\left(c_{j+m}\right)^{p} 2^{-(k n+j+m)} \\
& =2^{l} d_{p, m} \sum_{k=q}^{\infty}\left[\left(2^{n} s_{1} \ldots s_{n}\right)^{p} 2^{-n}\right]^{k},
\end{aligned}
$$

where

$$
d_{p, m}=\sum_{j=1}^{n}\left(c_{j+m}\right)^{p} 2^{-(j+m)} .
$$

The series (4.5) converges if and only if $2^{n} s_{1} \ldots s_{n}<2^{n / p}$; this is condition (4.4).

The mean of $\omega$ on $J_{l}$ is

$$
\frac{1}{\left|J_{l}\right|} \int_{J_{l}} \omega=2^{l} d_{1, m} \sum_{k=q}^{\infty}\left(s_{1} \ldots s_{n}\right)^{k}
$$

this series converges since each $s_{i}$ is less than one.

If $2^{n} s_{1} \ldots s_{n}<2^{n / p}$, then on summing the series in (4.5) and (4.6) we find that

$$
\frac{\frac{1}{\left|J_{l}\right|} \int_{J_{l}} \omega^{p}}{\left(\frac{1}{\left|J_{l}\right|} \int_{J_{l}} \omega\right)^{p}}=2^{l(1-p)} \frac{d_{p, m}}{\left(d_{1, m}\right)^{p}} 2^{n(p-1) q} \frac{\left[1-\left(s_{1} \ldots s_{n}\right)\right]^{p}}{1-\left(2^{n} s_{1} \ldots s_{n}\right)^{p} 2^{-n}},
$$

for all $l=q n+m \geq 0$ with $q \geq 0$ and $0 \leq m \leq n-1$. This expression depends on $p, n$, and $m$ but is independent of $q$, since $d_{p, m}$ and $d_{1, m}$ are independent of $q$ and the exponent of 2 in the right hand side of (4.7) reduces to $m(1-p)$. Taking the maximum over $m \in\{0, \ldots, n-1\}$, we see that there is a uniform upper bound, depending only on $p$ and $n$, for $\frac{1}{\left|J_{l}\right|} \int_{J_{l}} \omega^{p} /\left(\frac{1}{\left|J_{l}\right|} \int_{J_{l}} \omega\right)^{p}$, for all $l \geq 0$. Therefore $\omega \in \mathbf{R H}_{\mathbf{p}}^{\mathbf{d}}$, which establishes the claim.

Let $f\left(x_{1}, \ldots, x_{n}\right)=2^{n} x_{1} \ldots x_{n}$. Then by the claim above, the $n$-periodic weight $\omega$ associated to the point $\left(s_{1}, \ldots, s_{n}\right)$ in the open unit $n$-cube $(0,1)^{n}$ is in $\mathbf{R H}_{\mathbf{p}}^{\mathbf{d}}$ if and only if $f\left(s_{1}, \ldots, s_{n}\right)<2^{n / p}$.

By Lemma 2.4, the $\lambda$-operation takes the weight $\omega$ with sequence $\left\{s_{i}\right\}$ to a new weight $\omega_{\lambda}$ with sequence $\left\{s_{i}(\lambda)\right\}$, where

$$
s_{i}(\lambda)=\frac{1}{2}+\lambda\left(s_{i}-\frac{1}{2}\right) .
$$

In the unit $n$-cube, the $\lambda$-operation moves the point $P:\left(s_{1}, \ldots, s_{n}\right)$ towards the centre $Q:(1 / 2, \ldots, 1 / 2)$ of the cube along the line segment $L$ joining $P$ to $Q$. For $\lambda \in(0,1)$, the point $P_{\lambda}:\left(s_{1}(\lambda), \ldots, s_{n}(\lambda)\right)$ associated to $\omega_{\lambda}$ lies on $L$ between $P$ and $Q$.

We wish to find a weight $\omega$ and a number $\lambda \in(0,1)$ such that $\omega \in \mathbf{R H}_{\mathbf{p}}^{\mathbf{d}}$ but $\omega_{\lambda} \notin \mathbf{R} \mathbf{H}_{\mathbf{p}}^{\mathbf{d}}$. To do this, we will exhibit a line $L$ through $Q$, and points $P$ and $P_{\lambda}$ 
on $L$, such that: $(i) P$ and $P_{\lambda}$ are inside the open unit cube $(0,1)^{n}$, and $P_{\lambda}$ is between $P$ and $Q$; $(i i)$ the value of $f$ at $P$ is strictly less than $2^{n / p}$; and (iii) the value of $f$ at $P_{\lambda}$ is at least $2^{n / p}$. Then: $(i)^{\prime}$ there is a unique $\lambda \in(0,1)$ such that the $\lambda$-operation takes the weight $\omega$ associated to $P$ to the weight $\omega_{\lambda}$ associated to $P_{\lambda}$; $(\text { ii })^{\prime}$ the weight $\omega$ associated to $P$ is in $\mathbf{R} \mathbf{H}_{\mathbf{p}}^{\mathbf{d}}$; and $(i i i)^{\prime}$ the weight $\omega_{\lambda}$ associated to $P_{\lambda}$ is not in $\mathbf{R H}_{\mathbf{p}}^{\mathbf{d}}$.

Fix $a \in(0,1 / 2)$. Let $L$ be the line through $(a, 1, \ldots, 1)$ and $Q$; it has equation $\left(a+\left(\frac{1}{2}-a\right) t, 1-\frac{t}{2}, \ldots, 1-\frac{t}{2}\right)$, and values of $t \in[0,2]$ correspond to points in the closed unit cube $[0,1]^{n}$. Let $g(t)$ be the restriction of $f$ to $L$, for $t \in[0,2]$ :

$$
g(t)=(2 a+(1-2 a) t)(2-t)^{n-1} .
$$

Then $g^{\prime}(t)=(2-t)^{n-2}[-n(1-2 a) t+2(1-(n+1) a)]$.

If $n>2, g$ has two critical points; if $n=2, g$ has a single critical point. Since $g(2)=f(1-2 a, 0, \ldots, 0)=0$, and $f$ is positive in the open unit cube, a critical point of $g$ at $t=2$ is a minimum. The critical point which occurs at

$$
t_{m}=\frac{2(1-(n+1) a)}{n(1-2 a)}
$$

is a maximum for $g$. Denote this point by $P_{m}$. The maximum value is

$$
g\left(t_{m}\right)=\frac{2^{n}(n-1)^{n-1}(1-a)^{n}}{n^{n}(1-2 a)^{n-1}} .
$$

This maximum is taken inside the open unit cube if and only if $t_{m}>0$; in other words, if and only if $a<(n+1)^{-1}$. In this case, $P_{m}$ lies between $(a, 1, \ldots, 1)$ and the centre $Q$ of the cube.

The maximum value of $g$ is a continuous, increasing function of $a$, for $a \in$ $\left[0,(n+1)^{-1}\right]$. We denote this function by $h(a)=g\left(t_{m}\right)$ (see (4.11) above). Then $h^{\prime}(a)>0$ for $a \in\left[0,(n+1)^{-1}\right]$. Also, $h(0)=2^{n}(n-1)^{n-1} n^{-n}$, and $h\left(\frac{1}{n+1}\right)=\frac{2^{n}}{n+1}$. Therefore, if $p>1$ is large enough that

$$
2^{n / p}<\frac{2^{n}}{n+1}
$$

then we can choose $a_{p} \in\left(0,(n+1)^{-1}\right)$ so that $h\left(a_{p}\right) \geq 2^{n / p}$ and so that the maximum value $h\left(a_{p}\right)$ of $f$ along the line segment from $\left(a_{p}, 1, \ldots, 1\right)$ to $Q$ is taken on at a point inside the open unit cube.

Notice that if $p$ is too large, we may not be able to find a line segment on which the maximum value $h\left(a_{p}\right)$ of $f$ is actually equal to $2^{n / p}$, since the smallest $h\left(a_{p}\right)$ is $h(0)$, which is strictly larger than one (if $n>2$ ).

Define $P_{\lambda}$ as follows: if $h(0)<2^{n / p}<\frac{2^{n}}{n+1}$, choose the unique $a_{p} \in\left(0,(n+1)^{-1}\right)$ such that $h\left(a_{p}\right)=2^{n / p}$, and let $P_{\lambda}=P_{m}$ for that $a_{p}$. If $2^{n / p} \leq h(0)$, choose $a_{p}$ small enough that $f\left(a_{p}, 1, \ldots, 1\right)=2^{n} a_{p}<2^{n / p}$, and let $P_{\lambda}=P_{m}$ for that $a_{p}$.

In both cases, $f\left(a_{p}, 1, \ldots, 1\right)<2^{n / p}$, while the value of $f$ at $P_{m}$ is at least $2^{n / p}$. Since $g$ is increasing for $0<t<t_{m}$, we may choose a point $P$ between $\left(a_{p}, 1, \ldots, 1\right)$ and $P_{\lambda}$ such that the value of $f$ at $P$ is strictly less than $2^{n / p}$. Let $\lambda$ be the unique number in $(0,1)$ such that the $\lambda$-operation takes this point $P$ to $P_{\lambda}$.

The weights $\omega$ and $\omega_{\lambda}$ associated to $P$ and $P_{\lambda}$ respectively, together with the number $\lambda$, furnish our example: $\omega$ is in $\mathbf{R} \mathbf{H}_{\mathbf{p}}^{\mathbf{d}}$ but $\omega_{\lambda}$ is not in $\mathbf{R} \mathbf{H}_{\mathbf{p}}^{\mathbf{d}}$. 
The critical value of $p$, from (4.12), is

$$
p_{c}=\frac{n \log 2}{n \log 2-\log (n+1)} .
$$

Examples for all $p>p_{c}$ can be found among the $n$-periodic weights of the form described above. Letting $n$ tend to infinity, we see that $p_{c}$ tends to 1 , and so we have found examples for all $p>1$.

\section{REFERENCES}

[B] S. Buckley, Summation conditions on weights. Michigan Math. J. 40 \#1, 153-170 (1993). MR 94d:42021

[Ch] M. Christ, Lectures on singular integral operators. Regional Conference Series in Math, AMS \# 77 (1990). MR 92f:42021

[D] G. David, Wavelets and singular integrals on curves and surfaces. Springer Verlag Lecture Notes in Math. \# 1465 (1991). MR 92k:42021

[FKP] R. Fefferman, C. Kenig, J. Pipher, The theory of weights and the Dirichlet problem for elliptic equations. Annals of Math. \# 134, 65-124 (1991). MR 93h:31010

[GC-RF] J. Garcia-Cuerva, J.L. Rubio de Francia, Weighted norm inequalities and related topics. North Holland (1985). MR 87d:42023

[H] A. Haar, Zur Theorie der orthogonalen Funktionensysteme. Math. Ann. \# 69, 331-371 (1910).

[JN] R. Johnson, C. J. Neugebauer, Homeomorphisms preserving $\mathbf{A}_{\mathbf{p}}^{\mathbf{d}}$. Revista Matemática Iberoamericana Vol. 3, No. 2, 249-273 (1987). MR 90d:42013

[M] Y. Meyer, Ondelettes et Opérateurs II. Herman (1990). MR 93i: 42003

[P1] M. C. Pereyra, On the resolvents of dyadic paraproducts. Revista Matemática Iberoamericana Vol. 10 No. 3, 627-664 (1994). MR 96e:42021

[P2] M. C. Pereyra, On the resolvent of the dyadic paraproduct, and a nonlinear operation on $\mathrm{RH}_{p}$ weights. Cont. Math. of AMS. Vol 189, 461-471 (1995). MR 96h:42016

Department of Mathematics and Statistics, University of New Mexico, Albuquerque, New MeXico 87131-1141

E-mail address: crisp@math.unm.edu

Department of Mathematics, Harvey Mudd College, Claremont, California 91711

E-mail address: lesley@math.rice.edu 